\title{
Programmed Death-Ligand I Expression in Breast Cancer Patients: Clinicopathological Associations from a Single-Institution Study
}

\author{
Nehad M Ayoub (D) \\ Mona Fares' \\ Raya Marji $\mathbb{D}^{2}$ \\ Samir M Al Bashir ${ }^{2}$ \\ Rami J Yaghan (D) ${ }^{3}$ \\ 'Department of Clinical Pharmacy, \\ Faculty of Pharmacy, Jordan University of \\ Science and Technology (JUST), Irbid, \\ 22I I0, Jordan; ${ }^{2}$ Department of Pathology \\ and Microbiology, Faculty of Medicine, \\ Jordan University of Science and \\ Technology, Irbid, 22 I I0, Jordan; \\ ${ }^{3}$ Department of Surgery, College of \\ Medicine and Medical Sciences, Arabian \\ Gulf University, Manama, Bahrain
}

\begin{abstract}
Purpose: Tumor expression of programmed death-ligand 1 (PD-L1) is associated with evasion of immune response in several types of malignancies and such expression may render patients eligible for PD-L1 inhibitors. The use of immune checkpoint blockade therapy has been recently approved for the treatment of breast cancer. However, PD-L1 expression data are lacking among Jordanian breast cancer patients. In this study, the tumor PD-L1 expression was characterized in breast cancer patients to assess their eligibility for immune checkpoint blockade therapy. The study also aimed to explore the association between tumoral PD-L1 expression and the clinicopathologic characteristics and the prognostic factors in patients with breast cancer.
\end{abstract}

Patients and Methods: Tissue samples were available from 153 female patients with primary invasive breast cancer. Immunohistochemistry was performed on paraffin-embedded tumor sections that were stained with a PD-L1 antibody. Expression of tumor PD-L1 was correlated with demographics, clinicopathologic characteristics, and prognosis.

Results: The mean age at diagnosis was $54.2 \pm 12.8$ years (median 52, interquartile range 45 65). The percentage of PD-L1-positive tumors was 26.1\%. PD-L1 expression on tumor cells significantly and positively correlated with tumor size ( $\mathrm{rho}=0.174, \mathrm{p}=0.032)$. PD-L1 positivity was significantly associated with the grade of carcinoma $(p=0.001)$, HER2-positivity $(\mathrm{p}=0.015)$, and lymphovascular invasion $(\mathrm{p}=0.036)$. PD-L1 intensity was significantly associated with tumor stage $(\mathrm{p}=0.046)$. No significant associations were observed for the PD-L1 expression status or intensity with patient menopausal status, hormone receptor expression, and molecular subtypes. PD-L1 expression significantly correlated with a worse prognosis of breast cancer patients at the time of diagnosis ( $r h o=0.230, \mathrm{p}=0.005$ ).

Conclusion: Tumor PD-L1 expression was associated with advanced clinicopathologic features and worse prognosis in this cohort of Jordanian breast cancer patients. Future studies are needed to better understand the impact of PD-L1 blockade therapy on treatment outcomes in eligible breast cancer patients in Jordan.

Keywords: breast cancer, PD-L1, immunohistochemistry, clinicopathologic, prognosis

\section{Introduction}

Cancer cells are characterized by their ability to escape the immune response through multiple tumor-mediated escape mechanisms. ${ }^{1,2}$ Tumors evade immune surveillance through immunoediting that allows the selection of tumor variants resistant to immune effectors as well as the establishment of an immunesuppressive status within the tumor microenvironment. ${ }^{1,2}$ The evasion of the immune system by the tumor is mediated by different mechanisms. The utilization
Correspondence: Nehad M Ayoub Department of Clinical Pharmacy, Faculty of Pharmacy, Jordan University of Science and Technology (JUST), Irbid, 221 I0, Jordan

Tel +962-2-7201000 Ext. 23809

Fax +962-2-7095। 23

Email nmayoub@just.edu.jo 
of immune checkpoints to suppress the cell-mediated immune response and further establish a status of immune tolerance is currently identified to be a key immune evasion mechanism in cancer cells. ${ }^{2}$ Immune checkpoints are inhibitory immunoreceptors that act mainly to maintain self-tolerance and avoid overstimulation of immune response. $^{3,4}$ Several immune checkpoints have been identified in cancers, however, of major importance are the programmed death-1 (PD-1) and its ligand, programmed death-ligand 1 (PD-L1). ${ }^{3,4}$ The binding of PD-1 to PD-L1 blocks $\mathrm{T}$ cell function and activation. ${ }^{2,5}$ Under normal conditions, the activation of the PD-1/PD-L1 pathway prevents overt immune response and maintains tolerance to self-antigens. ${ }^{6}$ Nevertheless, tumoral PD-L1 expression is associated with diminished immune response in the tumor microenvironment. ${ }^{6}$ PD-L1 is overexpressed on tumor cells in various solid cancers including lung cancer, $^{7}$ renal cell carcinoma, ${ }^{8}$ glioblastoma, ${ }^{9}$ melanoma, ${ }^{10}$ bladder cancer, ${ }^{11}$ colorectal cancer, ${ }^{12}$ and breast cancer. ${ }^{13,14}$ Although several studies have described the expression of PD-1 and PD-L1 in breast cancer patients, substantial discrepancies exist in the expression rates and the prognostic impact. Noda et al reported significantly greater PD-1 mRNA expression in tumor tissues of breast cancer patients compared to normal tissues. ${ }^{15}$ Besides, mRNA expression of PD-1 in peripheral blood was higher in breast cancer patients compared to healthy individuals. In term of prognostic impact, poor overall survival (OS) was associated with reduced PD-1 mRNA expression in tumor tissue as well as increased PD-1 expression in peripheral blood of breast cancer patients. ${ }^{15}$ Nevertheless, in another study, no difference was shown for the levels of PD-1-positive $\mathrm{T}$ cells in the peripheral blood of breast cancer patients among different disease stages or molecular subtypes compared to the healthy controls. ${ }^{16}$ The rate of $\mathrm{PD}$-1-positive tumor-infiltrating lymphocytes was reported in 104 out of 660 breast cancer cases $(15.8 \%){ }^{17}$ PD-1 positivity was associated with tumor size, grade, lymph node status, and worse OS in the luminal B and the basal-like subtypes. ${ }^{17}$ Similarly, Vidula et al revealed that PD-1 expression was higher in human epidermal growth factor receptor 2 (HER2)positive and triple-negative breast cancer (TNBC) molecular subtypes and was further associated with advanced tumor grade and pathologic complete response. ${ }^{18}$ Alternatively, a recent study showed that PD-1 protein expression was associated with improved OS in a cohort of 564 patients with early breast cancer. ${ }^{19}$ The expression of PD-L1 in breast tumors has been largely associated with unfavorable clinicopathologic features. In a systematic analysis of 47 studies by Huang et al, PD-L1 positivity was associated with large tumor size, high grade tumors, high Ki-67, and triple-negative subtype in patients with primary breast cancer. ${ }^{20}$ Muenst et al showed that PD-L1 was expressed in 152 of the 650 breast cancer tissues investigated (23.4\%). ${ }^{21}$ The expression was significantly associated with the age of patients, tumor size and grade, lymph node status, estrogen receptor (ER)-negative status, and high Ki-67 expression. ${ }^{21}$ However, the prognostic and/ or predictive impact for the expression of PD-L1 in breast cancer lacks consensus. $^{22}$ PD-L1 expression was a negative prognostic factor associated with a significantly worse OS in patients with luminal B, HER2-positive, and basal-like molecular subtypes. ${ }^{21}$ However, in other studies, PD-L1 expression was associated with improved therapeutic outcomes and prognosis in patients with breast cancer. ${ }^{23-26}$

Immune checkpoint inhibitors are a breakthrough in cancer therapy. ${ }^{27}$ Immune checkpoint blockade with antiPD-1/PD-L1 antibodies has been approved for the treatment of different types of cancers. ${ }^{3}$ In 2019 , the United States Food and Drug Administration (US FDA) approved atezolizumab (a PD-L1 inhibitor) in combination with protein-bound paclitaxel for the treatment of TNBC patients with an unresectable locally advanced or metastatic disease whose cells express PD-L1 as determined by an FDA-approved test. ${ }^{28}$ Additionally, the PD-1 inhibitor, pembrolizumab, was recently approved for the treatment of patients with locally recurrent unresectable or metastatic TNBC in combination with chemotherapeutic agents such as paclitaxel protein-bound, paclitaxel, or gemcitabine plus carboplatin. ${ }^{29}$ The introduction of these drugs to the treatment plans of breast cancer requires the evaluation of the expression of the immune checkpoints by cancer cells and the understanding of the immunogenicity of breast cancer in a specific population. Thus, the assessment of the PD-L1 expression status among Jordanian patients would be necessary to understand their eligibility for immune checkpoint inhibitors. Such expression data are lacking among Jordanian patients. In this study, we aimed to investigate the expression of PD-L1 in a cohort of Jordanian breast cancer patients. Also, to explore association between the expression of PD-L1 and the clinicopathologic characteristics and the prognostic factors among this cohort. 


\section{Materials and Methods}

\section{Patients and Tumor Samples}

One hundred and fifty-three adult female patients $(n=153)$ with a histologically confirmed diagnosis of primary invasive breast cancer were obtained from the archives of King Abdullah University Hospital (KAUH) through the period of 2014-2020. Patients who received neoadjuvant therapy were not included in this study. All patients were discussed in a dedicated tumor board before the commencement of treatment. Loco-regional control was achieved either by a modified radical mastectomy or conservation breast surgery with radiotherapy. Further chemotherapy, hormonal treatment, and/or targeted treatment were individualized according to receptor status and tumor board recommendations. The electronic database at KAUH was used to retrieve the demographic and anthropometric data of patients. According to the World Health Organization (WHO), body mass index (BMI) was calculated as the weight in kilograms divided by the square of the height in meters, and patients were classified as underweight, normal, overweight, and obese, based on the WHO classification system. ${ }^{30}$

Relevant tumor data were obtained from pathology reports issued by the Pathology Department for eligible patients at the time of diagnosis of breast cancer. Pathological data included the size of the tumor, histopathologic type, ipsilateral axillary lymph node status, the status of lymphovascular invasion (LVI), and the expression status of receptors (ER, progesterone receptor (PR), and HER2). The tumor-node-metastasis (TNM) stage was determined according to the American Joint Committee on Cancer, ${ }^{31}$ and the tumor grade was indicated based on the Nottingham Combined Histologic Grade system. ${ }^{32}$ Breast tumors were classified into a low grade (grade I), intermediate grade (grade II), and high grade (grade III) carcinomas. For HER2, negative expression was indicated by an immunohistochemistry (IHC) score of 0 or +1 whereas HER 2 overexpression was determined by a score of +3 . Fluorescence in situ hybridization analysis was used for equivocal results (score of +2 by IHC), and gene amplification was considered positive for HER2 overexpression. Four molecular subtypes of breast cancer were determined based on the expression of receptors, ${ }^{33}$ these are luminal $\mathrm{A}(\mathrm{ER}+$ and/or $\mathrm{PR}+$, HER2-), luminal B (ER+ and/or PR+, HER2+), HER2enriched (ER-, PR-, HER2+), and triple-negative (ER-, PR-, HER2-).
Table I Prognostic Score for Breast Cancer Patients

\begin{tabular}{|c|c|c|}
\hline $\begin{array}{l}\text { Prognostic } \\
\text { Factor }\end{array}$ & Points & Impact on Prognosis \\
\hline \multicolumn{2}{|l|}{ Age (years) } & \multirow{3}{*}{$\begin{array}{l}\text { Patients younger than } 35 \text { years of age } \\
\text { present with more aggressive tumors and } \\
\text { a worse prognosis }\end{array}$} \\
\hline$\geq 35$ & 0 & \\
\hline$<35$ & I & \\
\hline \multicolumn{2}{|l|}{ Tumor size $^{\dagger}$} & \multirow{4}{*}{$\begin{array}{l}\text { Larger tumor size is associated with } \\
\text { a reduced survival rate }\end{array}$} \\
\hline $\mathrm{TI}$ & 0 & \\
\hline T2 & I & \\
\hline T3 & 2 & \\
\hline \multicolumn{2}{|l|}{ Lymph nodes } & \multirow{3}{*}{$\begin{array}{l}\text { The presence of positive lymph nodes } \\
\text { highly influences the likelihood of } \\
\text { recurrence }\end{array}$} \\
\hline Negative & 0 & \\
\hline Positive & 1 & \\
\hline \multicolumn{2}{|l|}{ Tumor grade } & \multirow{4}{*}{$\begin{array}{l}\text { Increased tumor grade is associated with } \\
\text { higher rates of distant metastasis and } \\
\text { poorer survival }\end{array}$} \\
\hline I & 0 & \\
\hline II & I & \\
\hline III & 2 & \\
\hline \multicolumn{2}{|l|}{ LVI } & \multirow{3}{*}{$\begin{array}{l}\mathrm{LVI} \text { is a poor prognostic factor } \\
\text { representing the ability of the cancer cells } \\
\text { to spread via hematogenous routes }\end{array}$} \\
\hline $\begin{array}{c}\text { Not } \\
\text { identified }\end{array}$ & 0 & \\
\hline Identified & 1 & \\
\hline \multicolumn{2}{|l|}{ ER } & \multirow{2}{*}{$\begin{array}{l}\text { Expression of hormone receptors is } \\
\text { associated with higher response to } \\
\text { endocrine therapy and a longer disease- } \\
\text { free survival }\end{array}$} \\
\hline $\begin{array}{l}\text { Positive } \\
\text { Negative }\end{array}$ & $\begin{array}{l}0 \\
1\end{array}$ & \\
\hline \multicolumn{2}{|l|}{ PR } & \multirow{3}{*}{$\begin{array}{l}\text { Expression of hormone receptors is } \\
\text { associated with higher response to } \\
\text { endocrine therapy and a longer disease- } \\
\text { free survival }\end{array}$} \\
\hline Positive & 0 & \\
\hline Negative & 1 & \\
\hline \multicolumn{2}{|l|}{ HER2 } & \multirow{3}{*}{$\begin{array}{l}\text { Overexpression of HER2 is associated } \\
\text { with increased tumor aggressiveness, } \\
\text { recurrence rates, and mortality rates }\end{array}$} \\
\hline Negative & 0 & \\
\hline Positive & I & \\
\hline
\end{tabular}

Notes: The table has been adapted with the publisher's permission from Ayoub NM, Yaghan RJ, Abdo NM, Matalka II, Akhu-Zaheya LM, Al-Mohtaseb AH. Impact of Obesity on Clinicopathologic Characteristics and Disease Prognosis in Pre- and Postmenopausal Breast Cancer Patients: A Retrospective Institutional Study. J Obes. 2019;2019:3820759.35. TPatients with T4 tumors were excluded from prognostic score calculations.

Abbreviations: ER, estrogen receptor; HER2, human epidermal growth factor receptor 2; LVI, lymphovascular invasion; PR, progesterone receptor.

\section{Prognostic Factors and Prognostic Score}

A prognostic score was generated for each breast cancer patient by considering 8 prognostic factors based on the paper by Cianfrocca and Goldstein. ${ }^{34}$ Patient and tumor characteristics at diagnosis were included such as the age 
of the patient, primary tumor size, tumor grade, lymph node status, the status of LVI, and the expression status of hormone receptors and HER2. ${ }^{35}$ Each patient was given a score value for each of the prognostic factors and a final score was generated by summing the scores for all factors (Table 1). The prognostic score ranges from 0 to 10 , at which higher scores imply a worse prognosis. ${ }^{35}$

\section{Immunohistochemistry}

Formalin-fixed, paraffin-embedded tumor tissues were obtained from the Pathology Department, and IHC was performed on sections that were cut at a thickness of $3 \mu \mathrm{m}$. For the detection of PD-L1 (an immune checkpoint), the sections were heated for $1 \mathrm{hr}$ using an oven at $62^{\circ} \mathrm{C}$ and were let to cool down at room temperature. Afterward, the staining procedure was performed using the Ventana BenchMark ULTRA IHC/ISH fully automated staining system, ${ }^{36}$ followed by the standard immunohistochemical staining procedures of the Pathology laboratory. The primary PD-L1 antibody was added per manufacturer recommendations and the incubation time was 80 minutes (Clone 22C3, Dako, USA). Positive control slides were composed of tonsil tissue and negative controls were applied by replacing the primary antibody with a buffer.

\section{Evaluation of Immunostaining}

Two independent pathologists (RM and SA) evaluated immunostaining and were blind to patient demographic and pathologic data. Discrepancies were resolved by joint discussion. PD-L1 immunostaining was evaluated by scanning the whole tumor section by the pathologists who provided a percentage of PD-L1 tumor cell positivity along with the intensity of staining. PD-L1 staining intensity was scored as negative, mild, moderate, or strong staining intensity. To assess positive versus negative expression of PD-L1 in the tumor tissue, a cut-off point value of $1 \%$ was considered for this analysis based on the percentage of PD-L1 positive cells. ${ }^{29}$ All evaluations were performed avoiding areas with necrosis, folded tissue, suboptimal preservation, and technical artifacts. Representative images of tumor PD-L1 staining are shown in Figure 1.

\section{Statistical Analysis}

The IBM SPSS statistical package was used to perform data analysis (IBM Corp. Version 26.0. Armonk, NY, USA). Continuous variables are presented as the mean \pm standard deviation (SD) or the median and interquartile range (IQR). Categorical variables were displayed as

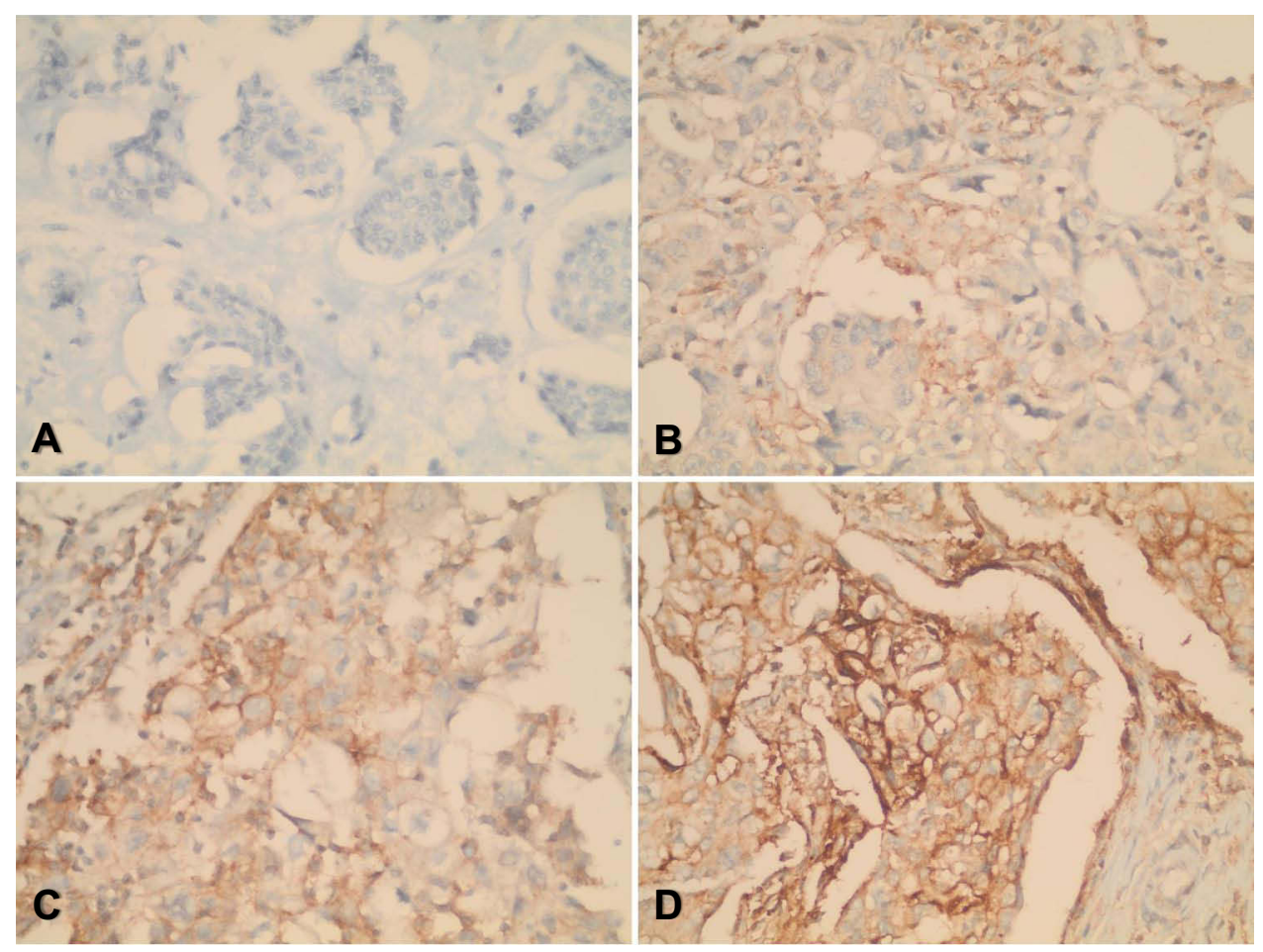

Figure I Immunohistochemistry staining for PD-LI in breast cancer tissues.

Notes: Representative images for (A) negative; (B) mild; (C) moderate; and (D) strong staining for PD-LI in tumor cells. [Magnification at 400x]. Abbreviation: PD-LI, programmed death-ligand I. 
frequency and percentages (n, \%). For group comparisons, the Mann-Whitney $U$-test and the Kruskal-Wallis analysis of variance were applied to compare two and multiple independent groups, respectively. Pearson's chi-square test of independence was used to assess associations between categorical variables and Spearman correlation test was applied to assess correlations between continuous variables. All p-values were two-sided and were statistically significant at $\mathrm{p}<0.05$.

Dichotomizing of some study variables was considered ahead of performing statistical analysis to avoid a small sample size upon further data analysis. ${ }^{37}$ Hence, the tumor stage was divided into early (I/II) and advanced (III/IV) and the grade was grouped into grades (I/II) and grade (III) breast cancer. The selection of these categories for variables was based on previously published cut points in the literature. $^{35,37}$

\section{Results}

\section{Demographic and Tumor Characteristics of Breast Cancer Patients}

The demographic and tumor characteristics of patients are shown in Table 2. The mean age at diagnosis was 54.2 \pm 12.8 years, ranging from 29 to 84 (median 52, IQR 4565 ). The average BMI at diagnosis was $30.5 \pm 5.9 \mathrm{~kg} / \mathrm{m}^{2}$, ranging from 16.8 to 46.9 (median 30.1, IQR 26.2-34) and approximately half of patients (53.4\%) were obese. A positive family history of breast cancer in first-degree relatives was reported in $25.2 \%$ of patients. Sixty-four patients (48.1\%) were premenopausal and 69 (51.9\%) were postmenopausal.

The average tumor size was $4.1 \pm 2.4 \mathrm{~cm}$ ranging from 1 to 20 (median 3.5, IQR 2.6-4.9). The mean number of involved lymph nodes was $5.6 \pm 7.8$ ranging from 0 to 38 (median 2, IQR 0-7). Stage II disease was indicated in $43.4 \%$ of patients and $51.3 \%$ of them had grade II carcinoma. Invasive ductal carcinoma was the most commonly reported histopathologic type (73.9\%) and luminal A was the most frequent molecular subtype (69.5\%). Other demographic and tumor characteristics are shown in Table 2 .

\section{Expression of PD-LI in Breast Cancer Tissues}

The percentage of PD-L1-positive tumors and the staining intensity are shown in Table 3 . The percentage of PD-L1positive tumors was $26.1 \%$. Only five patients $(3.3 \%)$ had
Table 2 Demographic and Tumor Characteristics of Breast Cancer Patients

\begin{tabular}{|c|c|}
\hline Characteristics & n (\%) \\
\hline \multicolumn{2}{|l|}{ Age, years } \\
\hline $18-39$ & $18(11.8)$ \\
\hline $40-59$ & $80(52.6)$ \\
\hline$\geq 60$ & 54 (35.5) \\
\hline \multicolumn{2}{|l|}{$\mathrm{BMI}^{\dagger}$} \\
\hline Underweight & $3(2.1)$ \\
\hline Normal weight & $22(15.1)$ \\
\hline Overweight & $43(29.5)$ \\
\hline Obese & $78(53.4)$ \\
\hline \multicolumn{2}{|l|}{ Marital status } \\
\hline Single & $9(6.1)$ \\
\hline Married & $135(9 \mid .8)$ \\
\hline Widowed & $\mathrm{I}(0.7)$ \\
\hline Divorced & $2(1.4)$ \\
\hline \multicolumn{2}{|l|}{ Family history of breast cancer in first-degree relatives } \\
\hline Present & $37(25.2)$ \\
\hline Absent & $110(74.8)$ \\
\hline \multicolumn{2}{|l|}{ Menopausal status } \\
\hline Premenopausal & $64(48.1)$ \\
\hline Postmenopausal & $69(51.9)$ \\
\hline \multicolumn{2}{|l|}{ Site } \\
\hline Right & $64(41.8)$ \\
\hline Left & $89(58.2)$ \\
\hline \multicolumn{2}{|l|}{ Tumor size } \\
\hline TI & $17(11.1)$ \\
\hline $\mathrm{T} 2$ & $97(63.4)$ \\
\hline T3 & $31(20.3)$ \\
\hline $\mathrm{T} 4$ & $8(5.2)$ \\
\hline \multicolumn{2}{|l|}{ Lymph node status } \\
\hline No & $42(27.6)$ \\
\hline NI & $45(29.6)$ \\
\hline N2 & $35(23)$ \\
\hline N3 & $30(19.7)$ \\
\hline \multicolumn{2}{|l|}{ TNM stage } \\
\hline I & $7(4.6)$ \\
\hline II & $66(43.4)$ \\
\hline III & $53(34.9)$ \\
\hline IV & $26(17.1)$ \\
\hline \multicolumn{2}{|l|}{ Grade } \\
\hline 1 & $17(\mid I .2)$ \\
\hline II & $78(5 \mathrm{I} .3)$ \\
\hline III & $57(37.5)$ \\
\hline \multicolumn{2}{|l|}{ Histologic type } \\
\hline Invasive ductal carcinoma & $113(73.9)$ \\
\hline Invasive lobular carcinoma & $10(6.5)$ \\
\hline
\end{tabular}

(Continued) 
Table 2 (Continued).

\begin{tabular}{|c|c|}
\hline Characteristics & n (\%) \\
\hline Mixed & $20(13.1)$ \\
\hline Other & $10(6.5)$ \\
\hline \multicolumn{2}{|l|}{ ER } \\
\hline Positive & I34 (88.7) \\
\hline Negative & I7 (II.3) \\
\hline \multicolumn{2}{|l|}{ PR } \\
\hline Positive & $125(81.7)$ \\
\hline Negative & $28(\mid 8.3)$ \\
\hline \multicolumn{2}{|l|}{ HER2 } \\
\hline Positive & $36(25.5)$ \\
\hline Negative & $105(74.5)$ \\
\hline \multicolumn{2}{|l|}{ LVI } \\
\hline Identified & 74 (49.7) \\
\hline Not identified & $75(50.3)$ \\
\hline \multicolumn{2}{|l|}{ Molecular subtype } \\
\hline Luminal A & $98(69.5)$ \\
\hline Luminal B & $29(20.6)$ \\
\hline HER2-enriched & $7(5.0)$ \\
\hline Triple-negative & $7(5.0)$ \\
\hline \multicolumn{2}{|l|}{ Surgery } \\
\hline Mastectomy & $139(90.8)$ \\
\hline Wide local excision & $12(7.8)$ \\
\hline Breast conservation & $2(1.3)$ \\
\hline Chemotherapy & $106(84.8)$ \\
\hline
\end{tabular}

Notes: ${ }^{\dagger}$ Patients were classified based on the World Health Organization (WHO) system for classification of obesity into underweight $\left(B M I<18.5 \mathrm{~kg} / \mathrm{m}^{2}\right)$, normal (BMI $18.5-24.99 \mathrm{~kg} / \mathrm{m}^{2}$ ), overweight (BMI $25.0-29.99 \mathrm{~kg} / \mathrm{m}^{2}$ ), and obese (BMI $\geq 30.0 \mathrm{~kg} /$ $\mathrm{m}^{2}$ ). Other histologic types included medullary, metaplastic, mucinous, and neuroendocrine carcinoma.

Abbreviations: BMI, body mass index; ER, estrogen receptor; HER2, human epidermal growth factor receptor 2; LVI, lymphovascular invasion; PR, progesterone receptor.

PD-L1 percentage positivity of $40 \%$ or greater. PD-L1 staining intensity was mild in 18 patients $(11.8 \%)$, moderate in 19 patients $(12.4 \%)$, and strong in 6 patients $(3.9 \%)$ (Table 3). When the data were stratified based on the molecular subtype, the percentage of PD-L1-positive staining was $21.4 \%$ in luminal $\mathrm{A}, 37.9 \%$ in luminal $\mathrm{B}$, $57.1 \%$ in HER2-enriched, and $14.3 \%$ in triple-negative tumors. The percentage of PD-L1-positive tumor cells was not significantly different among the molecular subtypes $(\mathrm{p}=0.111$, Kruskal-Wallis test).

The percentage of PD-L1-positive tumor cells significantly and positively correlated with primary tumor size in breast cancer patients ( $\mathrm{rho}=0.174, \mathrm{p}=0.032$, Table 4). No significant correlation was observed between PD-L1
Table 3 PD-LI Expression in Tumor Tissues of Breast Cancer Patients

\begin{tabular}{|l|c|}
\hline PD-LI Characteristics & n (\%) \\
\hline PD-LI expression status & \\
Positive & $40(26.1)$ \\
Negative & $113(73.9)$ \\
\hline Percentage of PD-LI-positive cells & \\
$<1 \%$ & $113(73.9)$ \\
I-9\% & $25(16.3)$ \\
I0-39\% & $10(6.5)$ \\
$\geq 40 \%$ & $5(3.3)$ \\
\hline Staining intensity of PD-LI-positive cells & \\
Negative & $110(71.9)$ \\
Mild & $18(11.8)$ \\
Moderate & $19(12.4)$ \\
Strong & $6(3.9)$ \\
\hline
\end{tabular}

Abbreviation: PD-LI, programmed death-ligand I.

expression and age of the patient at diagnosis, BMI, and the number of lymph nodes with detectable tumor cells (Table 4).

\section{Association of PD-LI Expression with Demographic and Tumor Characteristics of Breast Cancer Patients}

PD-L1 positivity was significantly associated with the grade of carcinoma $(p=0.001)$ (Table 5). PD-L1 was more positive in grade III $(60.0 \%)$ compared to grade I/II tumors (40.0\%). Furthermore, PD-L1-positive expression was significantly associated with both HER2-positivity and LVI $(p=0.015$ and $p=0.036$, respectively). Additionally, PD-L1 staining intensity was significantly associated with tumor grade and

Table 4 Correlation of PD-LI-Positive Tumor Cells with Demographic and Tumor Characteristics of Breast Cancer Patients

\begin{tabular}{|l|c|c|}
\hline \multirow{2}{*}{ Parameter } & \multicolumn{2}{|c|}{$\begin{array}{c}\text { Percentage of PD-LI-Positive } \\
\text { Tumor Cells }\end{array}$} \\
\cline { 2 - 3 } & Rho & P value \\
\hline Age, years & -0.110 & 0.177 \\
BMI, kg/m $\mathrm{m}^{2}$ & -0.063 & 0.451 \\
Tumor size, cm & 0.174 & $0.032^{*}$ \\
Number of lymph nodes & 0.101 & 0.216 \\
\hline
\end{tabular}

Notes: rho, Spearman correlation coefficient. *Indicates statistical significance at ${ }^{*} \mathrm{p}<0.05$.

Abbreviations: BMI, body mass index; PD-LI, programmed death-ligand I. 


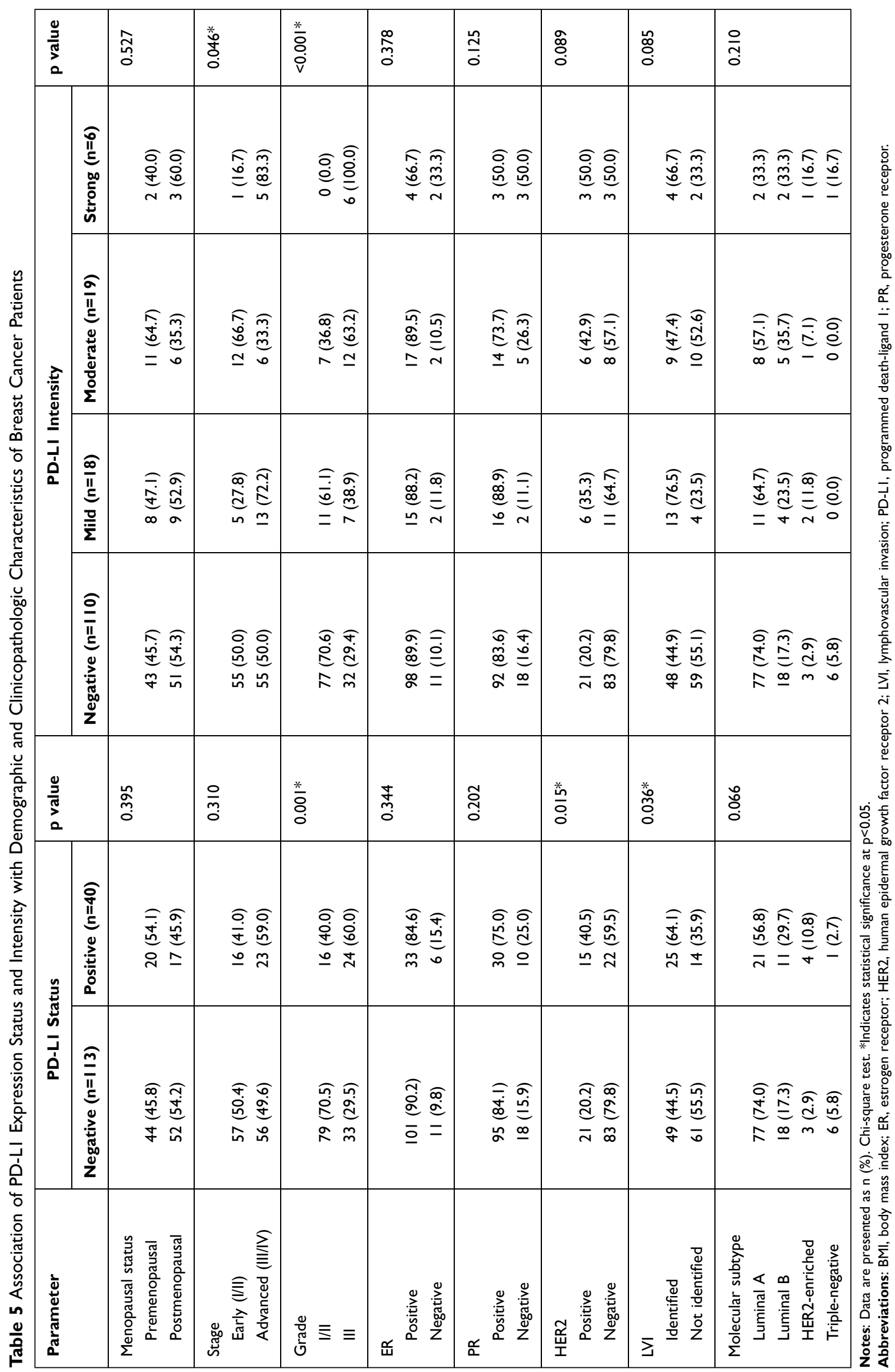




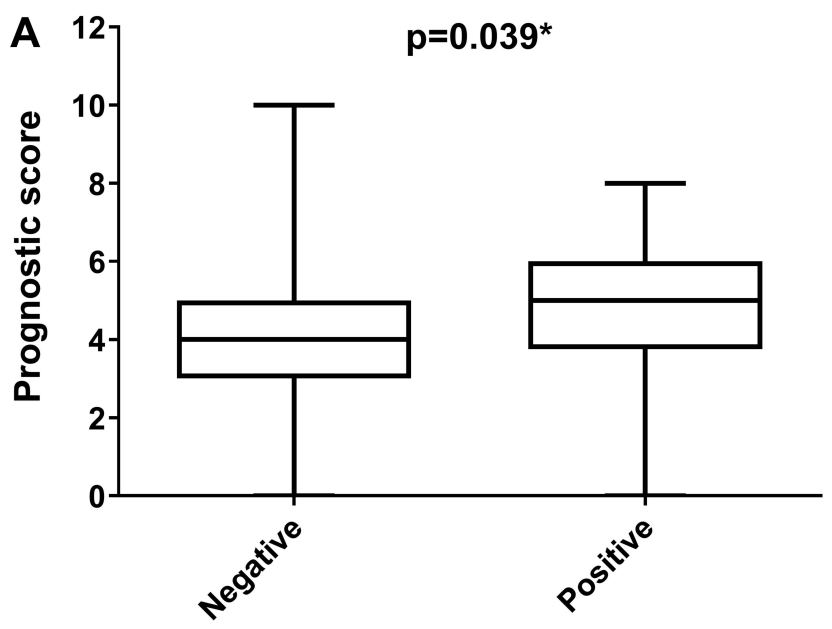

PD-L1 status

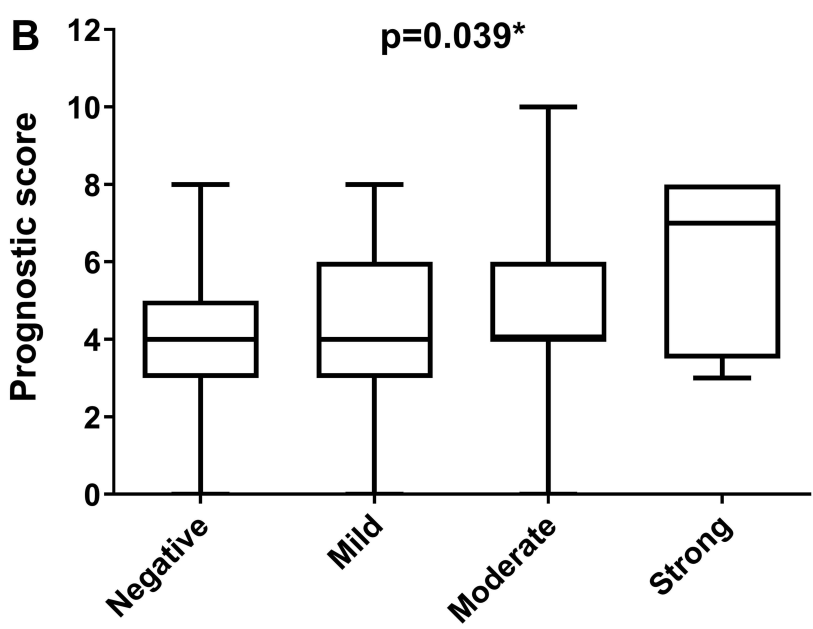

PD-L1 intensity

Figure 2 Prognostic scores in breast cancer patients based on PD-LI expression.

Notes: Prognostic scores according to (A) PD-LI expression status and (B) PD-LI staining intensity. Boxplots represent median prognostic scores. The bottom and top lines of the boxes represent the 25th and the 75 th percentiles, respectively, and the bars represent the minimum and maximum values. $*$ Indicates statistical significance at $\mathrm{P}<0.05$ according to Mann-Whitney U-test and Kruskal-Wallis test.

Abbreviation: PD-LI, programmed death-ligand I.

stage $(p<0.001$ and $p=0.046$, respectively $)$. In this regard, all patients who had strong PD-L1 staining intensity had grade III tumors and the majority $(83.3 \%)$ had advanced-stage disease. No significant associations were observed for the PD-L1 expression status and intensity with the menopausal status of patients, the expression status of hormone receptors, and molecular subtypes (Table 5).

\section{Impact of Tumoral PD-LI Expression on Prognosis of Breast Cancer Patients}

A prognostic score was generated for each patient using 8 prognostic factors. The mean prognostic score for breast cancer patients in this study was $4.1 \pm 1.8$, ranging from 0 to 10 (median 4, IQR 3-5). A significant positive correlation was found between the prognostic score and the percentage of PD-L1-positive tumor cells (rho $=0.230$, $\mathrm{p}=0.005)$. The median prognostic score was significantly different based on PD-L1 expression status $(\mathrm{p}=0.039)$ (Figure 2A). Patients with PD-L1-positive status had a significantly higher median of prognostic scores compared to patients with PD-L1-negative expression. Further, Kruskal-Wallis analysis revealed a significant difference in prognostic score among the PD-L1 staining intensity groups ( $p=0.039$, Figure 2B). Patients with strong PD-L1 staining intensity had a significantly higher prognostic score median compared to patients with negative staining.

\section{Discussion}

Breast cancer is the most common carcinoma among Jordanian females and the incidence of the disease is increasing annually. ${ }^{38}$ Recently, immune checkpoint inhibitors have been approved for several types of solid cancers and their indications are constantly expanding. Thus, the understanding of the expression landscape of target immune checkpoints is essential to delineate the status of antitumor immunity and further explore the eligibility of breast cancer patients for immunotherapy in Jordan. The fact that Jordanian patients with breast cancer have some different demographic characteristics compared to Western populations adds to the significance of studying such cohort of patients. These demographic characteristics are well demonstrated in our results and include: a younger mean age at diagnosis ( $54.2 \pm 12.8$ years), a high percentage of positive family history of breast cancer in firstdegree relatives (probably a reflection of the popularity of consanguineous marriage), and a high percentage of premenopausal breast cancer cases (48.1\%).

PD-L1 is the main ligand of PD-1 that is constitutively expressed in myeloid, lymphoid, and normal epithelial cells. $^{39}$ The binding of PD-1 to PD-L1 leads to the phosphorylation of the cytoplasmic region of PD-1 and the subsequent recruitment of phosphatases and downstream proteins such as spleen tyrosine kinase and phosphatidylinositol 3-kinase. ${ }^{6}$ Collectively, the activation of the PD-1/ 
Table 6 A Selected List for Studies Describing PD-LI Expression in Tumor Tissues of Female Breast Cancer Patients

\begin{tabular}{|l|l|l|l|l|}
\hline Reference Number & Number of Patients & Assay Applied & Percentage of PD-LI Positivity & Country/Population \\
\hline$[13]$ & 192 & TMA/IHC & $56.6 \%$ & Brazil \\
{$[14]$} & 245 & TMA/IHC & $12 \%$ & USA \\
{$[21]$} & 650 & TMA/IHC & $23.4 \%$ & Switzerland \\
{$[43]$} & Whole tissue/lHC & $20 \%$ & Greece \\
{$[44]$} & Moffitt tissue core/lHC & $21.7 \%$ & China \\
{$[47]$} & TMA/IHC & $32.8 \%$ & Middle East \\
{$[48]$} & TMA/IHC & $20.2 \%$ & Netherlands \\
{$[52]$} & Whole tissue/lHC & $33.1 \%$ & China \\
Current study & 1003 & Whole tissue/lHC & $26.1 \%$ & Jordan \\
\hline
\end{tabular}

Abbreviations: IHC, immunohistochemistry; PD-LI, programmed death-ligand I; TMA, tissue microarray.

PD-L1 pathway inhibits cytokine production and negatively regulates the immune function by inhibiting the activation and proliferation of $\mathrm{T}$ lymphocytes. ${ }^{6,40} \mathrm{In}$ the tumor microenvironment, the expression of PD-L1 on tumor cells promotes tumor cell immune evasion by delivering inhibitory signals to maintain immune tolerance. ${ }^{6,40}$ The expression of PD-L1 has been reported in breast cancer but not in normal breast tissue..$^{41}$ Lou et al indicated that PD-L1 was expressed in samples of invasive ductal breast carcinoma but not the adjacent normal breast tissue. ${ }^{42}$ In this study, we demonstrated a relatively low frequency of PD-L1 expression in Jordanian female breast cancer patients $(26.1 \%)$. Other studies showed a similar rate of PD-L1 positivity in patients with primary invasive breast cancer. ${ }^{43,44}$ Alternatively, Ali et al showed that PDL1 expression was detectable in $1.7 \%$ of breast tumor cells and was mostly expressed in basal-like tumors. ${ }^{45}$ In another study, only $4.1 \%$ of the invasive ductal carcinomas were PD-L1-positive. ${ }^{46}$ PD-L1 expression was higher in Middle Eastern and Brazilian breast cancer patients with expression rates of $32.8 \%$ and $56.6 \%$ of cases, respectively. ${ }^{13,47}$ PD-L1 expression rates vary widely as no standardized method for scoring is available in the meantime. Additionally, the differences in the cut-off values for PD-L1 positivity in clinical studies (1\% vs $5 \%)$ and the multiple primary antibodies available for staining are potential reasons for the variability in the rates of PD-L1 expression among the different studies. ${ }^{25,48,49}$ This discrepancy calls for the need to standardize staining and scoring methods to better understand and analyze the PD-L1 expression data among the different populations of breast cancer patients. ${ }^{48}$ Other potential causes for the discrepancies in the expression of PD-L1 can be explained by the variable populations of breast cancer patients examined, their varied demographics, and clinical characteristics. Table 6 summarizes findings from selected studies regarding the expression of PD-L1 in tumor tissues of female breast cancer patients.

An increasing number of clinical studies demonstrated the association of PD-L1 tumor expression with poor prognostic features and high-risk clinicopathological parameters in breast cancer patients. In this study, PD-L1 expression on tumor cells positively correlated with tumor size. Additionally, PD-L1-positivity was associated with grade III tumors, HER2-positivity, and LVI. These findings agree with several previous studies that have reported correlations between PD-L1 and unfavorable clinicopathological features, including larger tumor size, advanced grade, positive lymph nodes, LVI, and hormone receptor negativity. ${ }^{14,20,26,44,50}$ In a study by Hou et al, tumoral PD-L1 expression was reported in 17\% of HER2positive breast cancers analyzed and was positively associated with high tumor grade. ${ }^{51}$ In another study, an analysis of 126 patients with HER2-positive breast cancer indicated that $17.5 \%$ of patients were PD-L1 positive. ${ }^{23}$ PD-L1 expression was associated with response in patients who received neoadjuvant chemotherapy with the antiHER2 drug trastuzumab. In agreement, Hou et al indicated that PD-L1 expression correlated with better outcomes in patients with invasive HER2-positive tumors who are treated with a combination of chemotherapy and HER2targeted therapy. ${ }^{24}$ Other studies showed that intratumoral PD-L1 expression was significantly associated with the expression of hormone receptors and $\mathrm{Ki}-67 .{ }^{43,52}$ In a study by Qin et al, factors that were more likely associated with high PD-L1 expression in Chinese breast cancer patients included age younger than 35 years, advanced stage, larger tumor size, and LVI. ${ }^{44}$ In line with this, Parvathareddy et al showed that PD-L1 expression was associated with younger age, advanced grade, hormone 
receptors negativity, and triple-negative subtype in Middle Eastern breast cancer patients. ${ }^{47}$

Considering the heterogeneous nature of breast cancer, PD-L1 expression may vary among the different molecular subtypes. Both HER2-enriched and triple-negative molecular subtypes are known for their high mutational burden and are considered more immunogenic than luminal tumors. ${ }^{53}$ Previous studies demonstrated higher PD-L1 expression in HER2-enriched and TNBC compared to the luminal subtype. ${ }^{26,52,54}$ In our study, $90.1 \%$ of the tumors were of the luminal subtype which could explain the low rate of PD-L1 expression in the entire sample examined. However, the rate of PD-L1 positivity was highest in HER2-enriched tumors compared to other subtypes in our study. In contrast to these findings, Tsang et al showed that the expression rate of PD-L1 was highest in the luminal A subtype (34.1\%) and lowest in TNBC $(8.3 \%)$ among 1091 primary invasive breast cancers, and these differences were statistically significant. ${ }^{55}$ Furthermore, our results revealed a lack of association between PD-L1 expression and the molecular subtype of breast cancer. Such finding could be due to the relatively small sample size and reduced prevalence of non-luminal breast tumors in our study.

In this study, PD-L1 expression was adversely associated with prognostic features of breast cancer patients. Nevertheless, the impact of tumor PD-L1 expression on prognosis and survival of breast cancer patients generated conflicting results in the literature. While some studies described an association between PD-L1 expression and worse survival, others observed improved survival in PDL1 positive cases. In a meta-analysis involving 2061 patients, Guo et al reported that positive PD-L1 expression was a negative predictor for breast cancer as indicated by increased mortality risk and adverse clinicopathologic features. ${ }^{56}$ Moreover, PD-L1 positivity was associated with poor disease-free survival (DFS) and OS compared with PD-L1-negative expression. ${ }^{20,44}$ Alternatively, other studies indicated improved survival and outcomes in patients with basal-like and TNBC with positive PD-L1 expression. ${ }^{25,26}$ Recently, Kim et al showed that intratumoral PD-L1 expression was associated with better DFS and favorable outcomes in TNBC patients. ${ }^{54}$ Besides, PDL1 positivity was associated with longer DFS and OS compared with PD-L1-negative expression in a cohort of Chinese TNBC patients. ${ }^{57}$ Other studies, however, did not demonstrate a correlation between tumor PD-L1 expression and survival of breast cancer patients. ${ }^{49,58}$
The PD-1/PD-L1 pathway had emerged as a promising target for cancer therapy at which high-affinity anti-PD-1 or anti-PD-L1 monoclonal antibodies can reverse the immune tolerance and restore the activation and proliferation of the T cell response. ${ }^{5}$ The expression of PD-L1 on tumor cells has been used clinically to identify patients who are potential candidates for immunotherapy. In this regard, US FDA had approved atezolizumab, a PD-L1 inhibitor, in combination with chemotherapy for patients with metastatic TNBC who express PD-L1 on their tumor cells $(\geq 1 \%) .{ }^{59}$ Nevertheless, increasing evidence indicates that clinical benefit can be also achieved in cancer patients with negative tumor PD-L1 expression. In a meta-analysis by Shen and Zhao, data from 4174 patients with advanced or metastatic cancers from eight randomized controlled trials revealed that both PD-L1positive and PD-L1-negative patients responded to PD-L1 inhibitors and had significantly prolonged OS compared to conventional therapy. ${ }^{60}$ Similarly, a recent analysis by Liu et al demonstrated the efficacy of PD-1/PD-L1 inhibitors in both PD-L1-positive and PD-L1-negative breast cancer patients in terms of improving OS compared to controls. ${ }^{61}$ Collectively, these findings brought into question the value of PD-L1 expression status as the sole determinant for patients who are eligible to PD-L1 blockade therapy. ${ }^{60}$

This study has some limitations. First, the patient data was collected retrospectively. Second, the low number of breast tumors that correspond with the HER2-enriched and triple-negative tumors had hindered further analysis based on molecular subtype along with the potential to reveal associations with PD-L1 expression, if any. Third, the lack of recurrence and survival data was another limitation in this study. However, the major strengths of this study are the homogeneity of the population studied and the IHC assessment of whole tumor sections. Taking into consideration the substantial intratumoral heterogeneity in PD-L1 expression, ${ }^{14,48}$ we used entire tumor sections from patients' archival blocks to examine the expression of PDL1 in the whole tumor area to overcome the potential bias in scoring core biopsies.

\section{Conclusion}

The use of PD-1/PD-L1 checkpoint blockade therapy requires the understanding of the tumor microenvironment to identify patients who would potentially benefit from such therapy. To the best of our knowledge, this is the first study to characterize PD-L1 expression in female 
breast cancer patients in Jordan, which can be a model for the Arabian Middle East countries, since these countries share specific demographic characteristics. In this observational study, PD-L1 positivity correlated with unfavorable pathologic features and inferior prognosis in this sample of Jordanian breast cancer patients. Nevertheless, the expression of tumoral PD-L1 expression was not different among the molecular subtypes, in part, because of the under presentation of the HER2enriched and basal-like tumors in this cohort. Collectively, our findings call for future studies on a larger number of breast cancer patients to better assess the value of immunotherapeutic agents in treatment regimens for Jordanian breast cancer patients and to evaluate the impact of PD-1/PD-L1 inhibitors on treatment outcomes in eligible patients.

\section{Data Sharing Statement}

The data in this study are available from the corresponding author on reasonable request.

\section{Ethics Approval}

The Institutional Review Board (IRB) committee of the Jordan University of Science and Technology (JUST) and KAUH approved the study (Research number 14/126/2019). The study was conducted in concordance with the ethical principles of the Declaration of Helsinki. A consent form was not required by the IRB committee because of the retrospective and observational nature of the study that involved the use of archival tumor tissues. Data confidentiality was strictly maintained throughout the entire study, data were anonymized, and no patient identifiers were applied.

\section{Funding}

This work was supported by a grant from the Deanship of Research at the Jordan University of Science and Technology (JUST) (Research Grant No: 20190529).

\section{Disclosure}

The authors declare that they have no conflicts of interest.

\section{References}

1. Vinay DS, Ryan EP, Pawelec G, et al. Immune evasion in cancer: mechanistic basis and therapeutic strategies. Semin Cancer Biol. 2015;35(Suppl):S185-S198.

2. Ayoub NM, Al-Shami KM, Yaghan RJ. Immunotherapy for HER2-positive breast cancer: recent advances and combination therapeutic approaches. Breast Cancer (Dove Med Press). 2019;11:53-69.
3. He X, Xu C. Immune checkpoint signaling and cancer immunotherapy Cell Res. 2020;30(8):660-669. doi:10.1038/s41422-020-0343-4

4. Pardoll DM. The blockade of immune checkpoints in cancer immunotherapy. Nat Rev Cancer. 2012;12(4):252-264. doi:10.1038/ nrc3239

5. Xu-Monette ZY, Zhang M, Li J, Young KH. PD-1/PD-L1 blockade: have we found the key to unleash the antitumor immune response? Front Immunol. 2017;8:1597.

6. Setordzi P, Chang X, Liu Z, Wu Y, Zuo D. The recent advances of PD-1 and PD-L1 checkpoint signaling inhibition for breast cancer immunotherapy. Eur J Pharmacol. 2021;895:173867. doi:10.1016/j. ejphar.2021.173867

7. Yu H, Boyle TA, Zhou C, Rimm DL, Hirsch FR. PD-L1 expression in lung cancer. $J$ Thorac Oncol. 2016;11(7):964-975. doi:10.1016/j. jtho.2016.04.014

8. Choueiri TK, Fay AP, Gray KP, et al. PD-L1 expression in nonclear-cell renal cell carcinoma. Ann Oncol. 2014;25 (11):2178-2184. doi:10.1093/annonc/mdu445

9. Nduom EK, Wei J, Yaghi NK, et al. PD-L1 expression and prognostic impact in glioblastoma. Neuro Oncol. 2016;18(2):195-205. doi:10.1093/neuonc/nov172

10. Hino R, Kabashima K, Kato Y, et al. Tumor cell expression of programmed cell death-1 ligand 1 is a prognostic factor for malignant melanoma. Cancer. 2010;116(7):1757-1766. doi:10.1002/cncr.24899

11. Huang Y, Zhang SD, McCrudden C, Chan KW, Lin Y, Kwok HF. The prognostic significance of PD-L1 in bladder cancer. Oncol Rep. 2015;33(6):3075-3084. doi:10.3892/or.2015.3933

12. Li Y, Liang L, Dai W, et al. Prognostic impact of programed cell death-1 (PD-1) and PD-ligand 1 (PD-L1) expression in cancer cells and tumor infiltrating lymphocytes in colorectal cancer. Mol Cancer. 2016;15(1):55. doi:10.1186/s12943-016-0539-x

13. Baptista MZ, Sarian LO, Derchain SF, Pinto GA, Vassallo J. Prognostic significance of PD-L1 and PD-L2 in breast cancer. Hum Pathol. 2016;47(1):78-84. doi:10.1016/j.humpath.2015.09.006

14. Dill EA, Gru AA, Atkins KA, et al. PD-L1 expression and intratumoral heterogeneity across breast cancer subtypes and stages: an assessment of 245 primary and 40 metastatic tumors. Am J Surg Pathol. 2017;41(3):334-342. doi:10.1097/PAS.0000000000000780

15. Noda M, Masuda T, Ito S, et al. Circulating PD-1 mRNA in peripheral blood is a potential biomarker for predicting survival of breast cancer patients. Ann Surg Oncol. 2020;27(10):4035-4043. doi:10.1245/s10434-020-08375-Z

16. Du H, Yi Z, Wang L, Li Z, Niu B, Ren G. The co-expression characteristics of LAG3 and PD-1 on the T cells of patients with breast cancer reveal a new therapeutic strategy. Int Immunopharmacol. 2020;78:106113. doi:10.1016/j.intimp.2019.106113

17. Muenst S, Soysal SD, Gao F, Obermann EC, Oertli D, Gillanders WE. The presence of programmed death 1 (PD-1)positive tumor-infiltrating lymphocytes is associated with poor prognosis in human breast cancer. Breast Cancer Res Treat. 2013;139 (3):667-676. doi:10.1007/s10549-013-2581-3

18. Vidula N, Yau C, Rugo HS. Programmed cell death 1 (PD-1) receptor and programmed death ligand 1 (PD-L1) gene expression in primary breast cancer. Breast Cancer Res Treat. 2021;187(2):387-395. doi:10.1007/s10549-021-06234-3

19. Matikas A, Zerdes I, Lovrot J, et al. PD-1 protein and gene expression as prognostic factors in early breast cancer. ESMO Open. 2020;5 (6):e001032. doi:10.1136/esmoopen-2020-001032

20. Huang W, Ran R, Shao B, Li H. Prognostic and clinicopathological value of PD-L1 expression in primary breast cancer: a meta-analysis. Breast Cancer Res Treat. 2019;178(1):17-33. doi:10.1007/s10549019-05371-0

21. Muenst S, Schaerli AR, Gao F, et al. Expression of programmed death ligand 1 (PD-L1) is associated with poor prognosis in human breast cancer. Breast Cancer Res Treat. 2014;146(1):15-24. doi:10.1007/s10549-014-2988-5 
22. Matikas A, Zerdes I, Lovrot J, et al. Prognostic implications of PD-L1 expression in breast cancer: systematic review and meta-analysis of immunohistochemistry and pooled analysis of transcriptomic data. Clin Cancer Res. 2019;25(18):5717-5726. doi:10.1158/1078-0432.CCR-19-1131

23. Kurozumi $\mathrm{S}$, Inoue $\mathrm{K}$, Matsumoto $\mathrm{H}$, et al. Clinicopathological values of PD-L1 expression in HER2-positive breast cancer. Sci Rep. 2019;9(1):16662. doi:10.1038/s41598-019-52944-6

24. Hou Y, Nitta H, Wei L, et al. PD-L1 expression and CD8-positive $\mathrm{T}$ cells are associated with favorable survival in HER2-positive invasive breast cancer. Breast J. 2018;24(6):911-919. doi:10.1111/ tbj. 13112

25. Barrett MT, Lenkiewicz E, Malasi S, et al. The association of genomic lesions and PD-1/PD-L1 expression in resected triple-negative breast cancers. Breast Cancer Res. 2018;20(1):71. doi:10.1186/ s13058-018-1004-0

26. Sabatier R, Finetti P, Mamessier E, et al. Prognostic and predictive value of PDL1 expression in breast cancer. Oncotarget. 2015;6 (7):5449-5464. doi:10.18632/oncotarget.3216

27. Azoury SC, Straughan DM, Shukla V. Immune checkpoint inhibitors for cancer therapy: clinical efficacy and safety. Curr Cancer Drug Targets. 2015;15(6):452-462. doi:10.2174/ 156800961506150805145120

28. Narayan P, Wahby S, Gao JJ, et al. FDA approval summary: atezolizumab plus paclitaxel protein-bound for the treatment of patients with advanced or metastatic TNBC whose tumors express PD-L1. Clin Cancer Res. 2020;26(10):2284-2289. doi:10.1158/1078-0432. CCR-19-3545

29. FDA grants accelerated approval to pembrolizumab for locally recurrent unresectable or metastatic triple negative breast cancer. Available from: https://www.fda.gov/drugs/resources-informationapproved-drugs/fda-grants-accelerated-approval-pembrolizumablocally-recurrent-unresectable-or-metastatic-triple. Accessed July 5, 2021.

30. WHO Consultation on Obesity (1999: Geneva, Switzerland) \& World Health Organization. Obesity: Preventing and Managing the Global Epidemic: Report of a WHO Consultation. World Health Organization; 2000. Available from: https://apps.who.int/iris/handle/ 10665/42330. Accessed October 30, 2021.

31. Edge SB, Compton DR, Fritz CC, Greene AG, Trotti FA. AJCC Cancer Staging Handbook from the AJCC Cancer Staging Manual. 7th ed. New York, NY: Springer; 2010.

32. Rakha EA, Reis-Filho JS, Baehner F, et al. Breast cancer prognostic classification in the molecular era: the role of histological grade. Breast Cancer Res. 2010;12(4):207. doi:10.1186/bcr2607

33. Onitilo AA, Engel JM, Greenlee RT, Mukesh BN. Breast cancer subtypes based on ER/PR and Her2 expression: comparison of clinicopathologic features and survival. Clin Med Res. 2009;7(1-2):4-13. doi: $10.3121 / \mathrm{cmr} .2008 .825$

34. Cianfrocca M, Goldstein LJ. Prognostic and predictive factors in early-stage breast cancer. Oncologist. 2004;9(6):606-616. doi:10.1634/theoncologist.9-6-606

35. Ayoub NM, Yaghan RJ, Abdo NM, Matalka II, Akhu-Zaheya LM, Al-Mohtaseb AH. Impact of obesity on clinicopathologic characteristics and disease prognosis in pre- and postmenopausal breast cancer patients: a retrospective institutional study. $J$ Obes. 2019;2019:3820759. doi:10.1155/2019/3820759

36. Althammer S, Tan TH, Spitzmuller A, et al. Automated image analysis of NSCLC biopsies to predict response to anti-PD-L1 therapy. J Immunother Cancer. 2019;7(1):121. doi:10.1186/ s40425-019-0589-x

37. Stark A, Stahl MS, Kirchner HL, Krum S, Prichard J, Evans J. Body mass index at the time of diagnosis and the risk of advanced stages and poorly differentiated cancers of the breast: findings from a case-series study. Int J Obes (Lond). 2010;34(9):1381-1386. doi:10.1038/ijo.2010.69
38. Abdel-Razeq H, Mansour A, Jaddan D. Breast cancer care in Jordan. JCO Glob Oncol. 2020;6:260-268. doi:10.1200/JGO.19.00279

39. Kythreotou A, Siddique A, Mauri FA, Bower M, Pinato DJ. Pd-L1. J Clin Pathol. 2018;71(3):189-194. doi:10.1136/jclinpath-2017204853

40. Jia L, Zhang Q, Zhang R. PD-1/PD-L1 pathway blockade works as an effective and practical therapy for cancer immunotherapy. Cancer Biol Med. 2018;15(2):116-123. doi:10.20892/j.issn.20953941.2017 .0086

41. Li S, Chen L, Jiang J. Role of programmed cell death ligand-1 expression on prognostic and overall survival of breast cancer: a systematic review and meta-analysis. Medicine. 2019;98(16): e15201. doi:10.1097/MD.0000000000015201

42. Lou J, Zhou Y, Huang J, Qian X. Relationship between PD-L1 expression and clinical characteristics in patients with breast invasive ductal carcinoma. Open Med (Wars). 2017;12:288-292. doi:10.1515/ med-2017-0042

43. Evangelou Z, Papoudou-Bai A, Karpathiou G, et al. PD-L1 expression and tumor-infiltrating lymphocytes in breast cancer: clinicopathological analysis in women younger than 40 years old. In Vivo. 2020;34(2):639-647. doi:10.21873/invivo.11818

44. Qin T, Zeng YD, Qin G, et al. High PD-L1 expression was associated with poor prognosis in 870 Chinese patients with breast cancer. Oncotarget. 2015;6(32):33972-33981. doi:10.18632/ oncotarget. 5583

45. Ali HR, Glont SE, Blows FM, et al. PD-L1 protein expression in breast cancer is rare, enriched in basal-like tumours and associated with infiltrating lymphocytes. Ann Oncol. 2015;26(7):1488-1493. doi:10.1093/annonc/mdv192

46. Kassardjian A, Shintaku PI, Moatamed NA. Expression of immune checkpoint regulators, cytotoxic T lymphocyte antigen 4 (CTLA-4) and programmed death-ligand 1 (PD-L1), in female breast carcinomas. PLoS One. 2018;13(4):e0195958. doi:10.1371/journal. pone. 0195958

47. Parvathareddy SK, Siraj AK, Ahmed SO, et al. PD-L1 protein expression in middle eastern breast cancer predicts favorable outcome in triple-negative breast cancer. Cells. 2021;10:2. doi:10.3390/ cells 10020229

48. Manson QF, Ter Hoeve ND, Buerger H, Moelans CB, van Diest PJ. $\mathrm{PD}-1$ and PD-L1 expression in male breast cancer in comparison with female breast cancer. Target Oncol. 2018;13(6):769-777. doi:10.1007/s11523-018-0610-1

49. Mori H, Kubo M, Yamaguchi R, et al. The combination of PD-L1 expression and decreased tumor-infiltrating lymphocytes is associated with a poor prognosis in triple-negative breast cancer. Oncotarget. 2017;8(9):15584-15592. doi:10.18632/oncotarget.14698

50. Tawfik O, Kimler BF, Karnik T, Shehata P. Clinicopathological correlation of PD-L1 expression in primary and metastatic breast cancer and infiltrating immune cells. Hum Pathol. 2018;80:170-178. doi:10.1016/j.humpath.2018.06.008

51. Hou Y, Nitta H, Wei L, Banks PM, Parwani AV, Li Z. Evaluation of immune reaction and PD-L1 expression using multiplex immunohistochemistry in HER2-positive breast cancer: the association with response to anti-HER2 neoadjuvant therapy. Clin Breast Cancer. 2018;18(2):e237-e244. doi:10.1016/j.clbc.2017.11.001

52. Zhou T, Xu D, Tang B, et al. Expression of programmed death ligand- 1 and programmed death-1 in samples of invasive ductal carcinoma of the breast and its correlation with prognosis. Anticancer Drugs. 2018;29(9):904-910. doi:10.1097/ CAD. 0000000000000683

53. Criscitiello C, Curigliano G. Immunotherapy of Breast Cancer. Prog Tumor Res. 2015;42:30-43.

54. Kim HS, Do SI, Kim DH, Apple S. Clinicopathological and prognostic significance of programmed death Ligand 1 expression in Korean patients with triple-negative breast carcinoma. Anticancer Res. 2020;40(3):1487-1494. doi:10.21873/anticanres.14093 
55. Tsang JY, Au WL, Lo KY, et al. PD-L1 expression and tumor infiltrating PD-1+ lymphocytes associated with outcome in HER2+ breast cancer patients. Breast Cancer Res Treat. 2017;162(1):19-30. doi:10.1007/s10549-016-4095-2

56. Guo Y, Yu P, Liu Z, et al. Prognostic and clinicopathological value of programmed death Ligand-1 in breast cancer: a meta-analysis. PLoS One. 2016;11(5):e0156323. doi:10.1371/journal.pone.0156323

57. AiErken N, Shi HJ, Zhou Y, et al. High PD-L1 expression is closely associated with tumor-infiltrating lymphocytes and leads to good clinical outcomes in Chinese triple negative breast cancer patients. Int J Biol Sci. 2017;13(9):1172-1179. doi:10.7150/ijbs.20868

58. Brockhoff G, Seitz S, Weber F, et al. The presence of PD-1 positive tumor infiltrating lymphocytes in triple negative breast cancers is associated with a favorable outcome of disease. Oncotarget. 2018;9 (5):6201-6212. doi:10.18632/oncotarget.23717
59. Soare GR, Soare CA. Immunotherapy for breast cancer: first FDA approved regimen. Discoveries (Craiova). 2019;7(1):e91. doi:10.15190/d.2019.4

60. Shen X, Zhao B. Efficacy of PD-1 or PD-L1 inhibitors and PD-L1 expression status in cancer: meta-analysis. BMJ. 2018;362:k3529. doi:10.1136/bmj.k3529

61. Liu X, Guo CY, Tou FF, et al. Association of PD-L1 expression status with the efficacy of PD-1/PD-L1 inhibitors and overall survival in solid tumours: a systematic review and meta-analysis. Int $J$ Cancer. 2020;147(1):116-127. doi:10.1002/ijc.32744

\section{Publish your work in this journal}

Breast Cancer - Targets and Therapy is an international, peer-reviewed open access journal focusing on breast cancer research, identification of therapeutic targets and the optimal use of preventative and integrated treatment interventions to achieve improved outcomes, enhanced survival and quality of life for the cancer patient.
The manuscript management system is completely online and includes a very quick and fair peer-review system, which is all easy to use. Visit http://www.dovepress.com/testimonials.php to read real quotes from published authors. 